EPJ Web of Conferences 19, 10003 (2012)

DOI: $10.1051 /$ epjconf/20121910003

(C) Owned by the authors, published by EDP Sciences, 2012

\title{
Models of disk chemical evolution focusing the pure dynamical radial mixing
}

\author{
A. Curira , A. Spagna, M.G. Lattanzi, G. Murante, \\ and P. Re Fiorentin
}

INAF, Astronomical Observatory of Torino, Italy

\begin{abstract}
We performed N-body simulations to study the dynamical evolution of a stellar disk inside a Dark Matter (DM) halo. Our results evidence how a standard -radially decreasing- metallicity gradient produces a negative $v_{\phi}$ vs. $[\mathrm{Fe} / \mathrm{H}]$ correlation, similar to that shown by the thin disk stars, while an inverse radial gradient generates a positive rotation-metallicity correlation, as that observed in the old thick population.
\end{abstract}

\section{METHODS}

We used a halo with a cosmological radial density profile, mass, radius and concentration appropriate for a Milky Way-like DM halo. Embedded in our halo is an exponential stellar disk; two cases have been analyzed: a bulgeless disk and a disk endowed with a bulge. The bulge has an Hernquist radial density profile. The bulgeless disk develops a bar, whereas the disk with a bulge is stable. We investigate the impact of the bar structure on the stellar migration and on the formation of the thick disk. We also follow the redistribution of the metallicity inside the disk due to radial mixing. By using non-dissipative models, we investigate the contribution of pure dynamics to chemical evolution.

\section{RESULTS}

The particles from the inner regions of the disk migrate outward to higher $z$ (as shown in fig. 1). We concentrate our analysis on the annulus between 8 and $10 \mathrm{kpc}$, which is related to the solar neighborhood. We follow the chemical redistribution of an assigned metallicity gradient: fig. 2 shows the initial gradient throughout the disk taken from [1]. Such a gradient reproduces the actual chemical distribution inside the thin disk of the Milky Way. In fig. 2b we show as the dynamical evolution of such a gradient produces after $5 \mathrm{Gyr}$ a velocity-metallicity correlation similar to the one shown in [2] (fig. 2c, lower panel), as compared to that from the Geneva-Copenhagen Survey (fig. 2c, upper panel). In the case of an inverse initial gradient, as in fig. 3a (see [3]), the disk evolution produces the metallicity-velocity correlation for stars having height $|z|>1.5$ in fig. $3 \mathrm{~b}$. Such a correlation is in qualitative agreement with the one shown by Spagna et al in [4] (fig. 3c) for the thick disk stars.

\section{CONCLUSIONS}

Non dissipative diffusion mechanisms inside disks as theorized by Wielen [5] are very efficient to promote radial migration. According to Sellwood and Binney [6] non axisymmetric structures like spiral

\footnotetext{
ae-mail: curir@oato.inaf .it
}

This is an Open Access article distributed under the terms of the Creative Commons Attribution-Noncommercial License 3.0, which permits unrestricted use, distribution, and reproduction in any noncommercial medium, provided the original work is properly cited. 


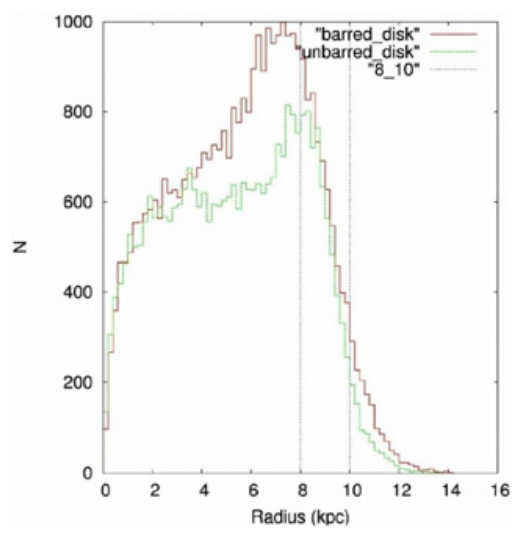

Figure 1. This diagram shows the original radii $(\mathrm{at} \mathrm{t}=0$ ) of the the particles that are at distance $z>1.5 \mathrm{kpc}$ from the disk equatorial plane after $\mathrm{t}=5 \mathrm{Gyr}$ of evolution. It is evident that the particles migrate from the inner radii toward larger radii and larger $z$ and that the peak shift away from the annulus between [8-10] kpc radii is bigger for a barred disk (red lines) than in a non barred one (green lines). Moreover the high $z$ region appears more populated at the end of the evolution in the barred case.
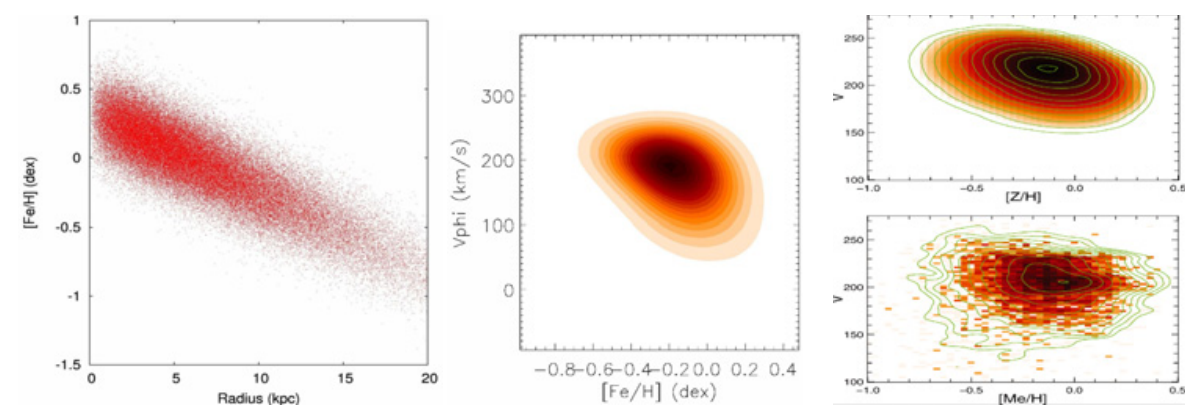

Figure 2. $a, b, c$ from left to right: $2 a$ is the metallicity gradient assigned to the disk in the initial configuration. $2 b$ is the correlation velocity-metallicity obtained after $5 \mathrm{Gyr}$ of evolution. $2 \mathrm{c}$ is the same correlation observed in the Geneva-Copenhagen Survey (upper panel) and reproduced in the model by Shönrich and Binney (lower panel).
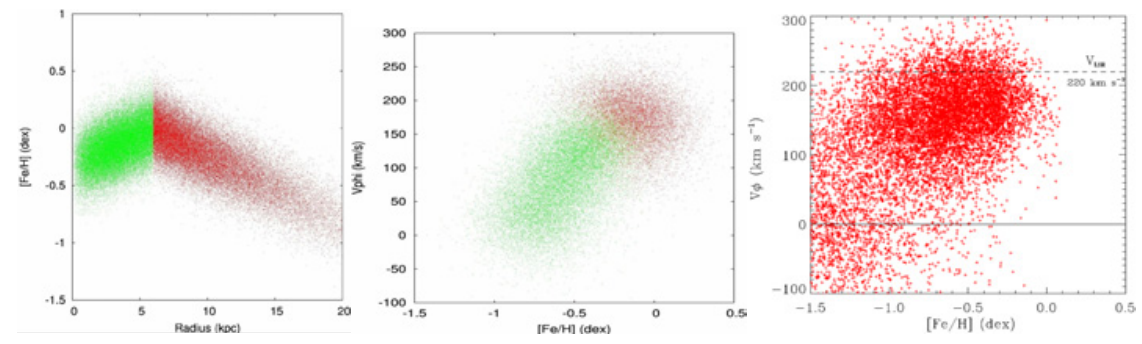

Figure 3. a,b,c from left to right: $3 \mathrm{a}$ is the early metallicity gradient plugged in the initial disk. $3 \mathrm{~b}$ is the final velocity-metallicity correlation obtained for the thick disk in this case. $3 \mathrm{c}$ is the same correlation observed by Spagna et al for stars having a distance from the disk plane greater than $1.5 \mathrm{kpc}$.

arms and bars should enhance this migration: the differences between our barred and non-barred models for disks confirm this effect. A correlation between the azimuthal velocity and the metallicity as in [4] can be produced by a pure dynamical migration if we assume an initial radial chemical gradient as suggested in [3] in their chemical evolution model for an early disk. 
Assembling the Puzzle of the Milky Way

\section{References}

[1] Chen et al IAU Symp 254 (2009) 15

[2] Shönrich, R. \& Binney, J. MNRAS (2009) 336, 785

[3] Chiappini, C. et al ApJ 554 (2001) 1044

[4] Spagna, A. et al A\&A (2010) 510, L4

[5] Wielen, R. A\&A (1977) 60, 263

[6] Sellwood, J. A. \& Binney, J. MNRAS (2002) 336,785 